\title{
The extinction law at high redshift
}

\section{Simona Gallerani*}

Scuola Normale Superiore

E-mail: simona.galleraniesns.it

\begin{abstract}
We analyze the optical-near infrared spectra of 33 quasars with redshifts $3.9<z<6.4$ with the aim of investigating the properties of dust extinction at these cosmic epochs. The Small Magellanic Cloud (SMC) extinction curve has been used to reproduce the dust reddening of most quasars at $z<2.2$; the main goal of this work is to investigate whether this curve provides a good prescription for describing dust extinction at higher redshifts or not.

We fit the observed spectra with synthetic absorbed quasar templates obtained by varying the intrinsic slope $(\alpha)$, the absolute extinction $\left(A_{3000}\right)$ and by using grid of empirical and theoretical extinction curves. We find that seven quasars in our sample require substantial extinction $\left(A_{3000}>0.8\right)$, and are characterized by very steep intrinsic slopes $(\alpha<-2.3)$. All of the individual quasars require extinction curves deviating from the SMC, with a tendency to flatten at lambda $<2000 \AA$ (rest frame). We obtain a mean extinction curve at $z>4$ by averaging the extinction curves inferred for individual quasars. With a confidence level exceeding $95 \%$, we can conclude that the extinction curve of BAL quasars deviates from that of the SMC. The different extinction curves in quasars at $z>4$ relative to quasars at lower redshift suggest either a different dust production mechanism at high redshift, or a different mechanism of processing dust into the interstellar medium (ISM).

We have applied the same method of analysis to the optical/near-infrared (IR) afterglow of GRB050904 at $z=6.3$. In this case, we find that the SMC extinction curve and a supernova-type one provide equally good fit to the data at all epochs, with an average amount of dust absorption at $\lambda_{\text {rest }}=3000 \AA$ of $A_{3000}=0.25 \pm 0.07$ mag. These results indicate both that the primeval galaxy at $z=6.3$ hosting this GRB has already enriched its ISM with dust, and that it is not possible to characterize the nature of dust suppliers in this source.
\end{abstract}

The Life Cycle of Dust in the Universe: Observations, Theory, and Laboratory Experiments - LCDU 2013, 18-22 November 2013

Taipei, Taiwan

\footnotetext{
*Speaker.
} 


\section{Evolution of dust properties through cosmic times}

Extensive measurements of the local interstellar extinction exist along hundreds of lines of sight in the Milky Way (MW) [1], and along tens of lines of sight in the Large Magellanic Cloud (LMC) and in the Small Magellanic Cloud (SMC) [2]. In particular, the extinction curve of the SMC is generally adopted also to describe the dust reddening of local QSOs [3-4] since the broad bump at $2175 \AA$, which is characteristic of the MW and LMC extinction curve, but absent in the SMC, has never been conclusively seen from quasar host galaxy dust. Recently, extinction curves have been also studied in several quasar and gamma ray bursts even at the highest redshifts probed so far [5-12]. These kind of studies allow to investigate whether the properties of dust evolve through cosmic times. Here, we briefly summarize the results obtained by Gallerani et al. [7] and Stratta et al. [9] and we discuss possible improvements for future investigations.

\section{Inferring dust extinction from quasar and gamma ray burst spectra}

To infer the properties of high-z dust we analyze a sample of optical and near-IR spectra of high-z quasars, observed with the TNG (Telescopio Nazionale Galileo), VLT, and GEMINI telescopes and of one gamma ray burst spectrum obtained with Swift. We fit the spectral region redward of the Ly $\alpha$ emission line with the following equation:

$$
F_{\lambda}=C F_{\lambda}^{t}\left(\frac{\lambda}{3000}\right)^{(1.62-\alpha)} 10^{-A_{3000} \frac{A_{\lambda} .5}{2.5}}
$$

where $\mathrm{C}$ is a normalization constant, $F_{\lambda}^{t}$ is a quasar template spectrum, $\alpha$ the slope of the unreddened spectrum, $A_{3000}$ the absolute extinction at $3000 \AA$, and $A_{\lambda}$ is the extinction curve normalized at $3000 \AA$. In this study we adopt the template by Reichard et al. [13], obtained considering 892 quasars classified as non-BAL. The spectral index of the averaged non-BAL spectrum $\left(F_{\lambda}^{t} \propto \lambda^{-\alpha_{t}}\right)$ is $\alpha_{t}=1.62$. Therefore, the term $(\lambda / 3000)^{(1.62-\alpha)}$ allows us to force the slope of the template to the value $\alpha$. We let $\alpha$ to vary in the interval $[0.2 ; 3.0]$, which is the range encompassed by more than $95 \%$ of the quasars. Concerning $A_{\lambda}$, we consider a grid of extinction curves to characterize the extinction produced by dust in the rest-frame wavelength range $0.1 \leq \lambda \leq 0.5 \mu \mathrm{m}$. First, we consider the empirical curves which describe the dust extinction in the local Universe [14]: the MW extinction curve, characterized by a a prominent bump at $2175 \AA$; the featureless SMC extinction curve, which steeply rises with inverse wavelength from near infrared to far ultraviolet $\left(A_{\lambda} / A_{3000} \sim \lambda^{-1.2}\right)$; and the LMC extinction curve, being intermediate between the MW and the SMC. We show the SMC, LMC and MW extinction curves in Figure 2, left upper panel (black solid, red dotted, green dashed lines, respectively). We also use the extinction curves expected by Type II SNe dust models as predicted by Todini \& Ferrara [15] and Bianchi \& Schneider [16] (left upper panel) as well as the results of the models by Hirashita et al. [17] for dust produced by SNe of progenitor mass $20 M_{\odot}$ (bottom left panel of Figure 2) and Pair Instability SNe (PISN) of progenitor mass of $170 M_{\odot}$ (bottom right panel). 

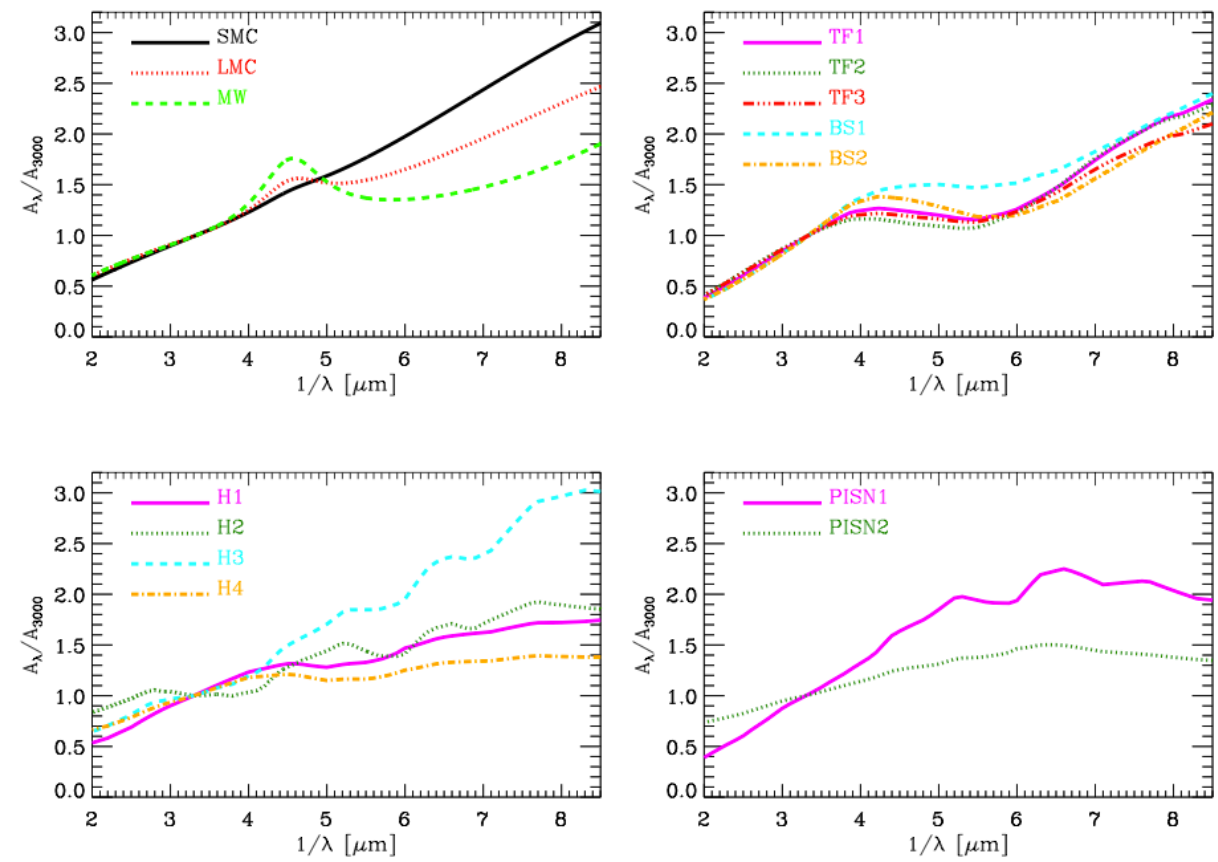

Figure 1: Upper left panel: Empirical curves for dust extinction in the local Universe. Theoretical extinction curves predicted by dust models of Type II SNe (upper right and bottom left panels) and pair instability SNe (bottom right panel).

\section{Observational constraint from quasar spectra at $3.9<z_{\mathrm{em}}<6.4$}

We apply the method described in the previous section to a sample of 33 observed quasars at $4 \leq z_{\text {em }} \leq 6.4$ to understand whether the SMC extinction curve is a good prescription for describing dust extinction at these redshifts. Observational data are from Juarez et al. [18], Jiang et al. [19], Willott et al. [20], and Mortlock et al. [21]. The $\chi^{2}$ analysis reveals that 7 quasars require substantial $\left(A_{3000} \geq 0.8\right)$ reddening and that these reddened spectra favor extinction curves which differ from the SMC (left panel in Fig. 2). The deviation is more promounced in the case of BAL quasars. BAL quasars are thought to be in an earlier evolutionary stage with respect to no-BAL quasars [22]. Therefore, we speculate that the extinction curve of high-z BAL quasars more properly reflect the dust properties at high redshift. For this reason, we compute the mean of the extinction curves inferred in the case of BAL to provide an empirical, average extinction law at $z \gtrsim 4$, called MEC, and shown in the right panel of Fig. 2. We find that the MEC deviates from the SMC extinction curve at a confidence level $\gtrsim 90 \%$ for $\lambda<2000 \AA$ in the rest frame of the source [7]. We finally comment on the fact that new Large Binocular Telescope observations obtained in the case of the most distant BAL quasar known (SDSS J1048+4637 at $\mathrm{z}=6.2$ ), confirm that the dust properties in this object are well reproduced by an SN-type extinction curve (Gallerani et al., in preparation).

\section{Observational constraint from a gamma-ray burst spectra at $z_{\mathrm{em}}=6.3$}

The only non-quasar host galaxy, with modest star formation rate, for which dust extinction 

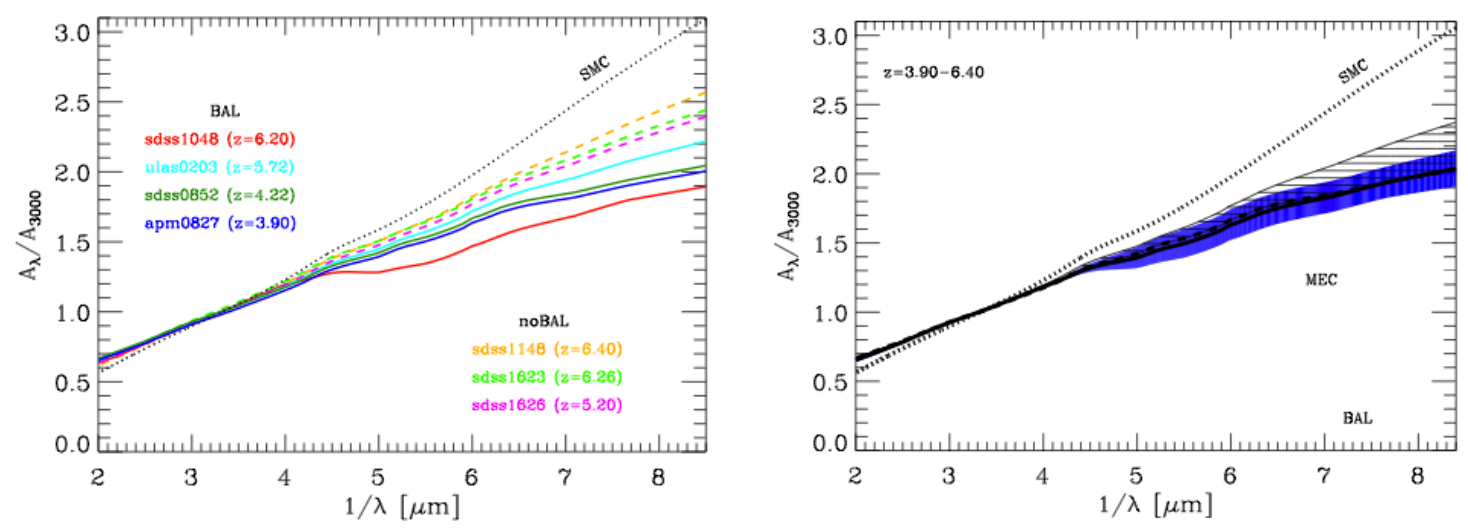

Figure 2: Left panel: Best fit extinction curves of reddened quasars. The solid lines are for BAL quasars, while dashed lines are for non-BAL quasars. For comparison, the SMC extinction curve is also shown and labeled in the figure (dotted black line). Right panel: The solid line shows the mean extinction curve (MEC) computed by averaging the results obtained for BAL reddened quasars, while the shaded region shows the dispersion. The dashed line shows the extinction curve obtained by the simultaneous fit of all the BAL quasars, while the hatched area shows the $68 \%$ confidence limit.

has been tentatively detected at $z \sim 6$ is the host of gamma ray burst GRB 050904 at $\mathrm{z}=6.3$ [9]. However, the claim of dust extinction for this GRB has been debated [10]. We suggest that the discrepant results occur primarily because most of previous studies have not simultaneously investigated the X-ray to near-IR spectral energy distribution of this GRB. The difficulty with this burst is that the X-ray afterglow is dominated by strong flares at early times and is poorly monitored at late times. In addition, the $\mathrm{Z}$ band photometry, which is the most sensitive to dust extinction, has been found to be affected by strong systematics. We have re-analyzed the Swift/XRT afterglow observations of this GRB, using extensive past studies of X-ray flare properties when computing the X-ray afterglow flux level and exploiting the recent reanalysis of the optical (UV rest frame) data of the same GRB. We extract the X-ray to optical/near-IR afterglow SED for the three epochs where the best spectral coverage is available: $0.47,1.25$, and 3.4 days after the trigger. A spectral power-law model has been fitted to the extracted SEDs. We have applied the same analysis adopted in the case of high-z quasars to GRB 050904. The results are shown in Fig. 3. In this case, the SMC extinction curve and the SN-type one provide equally good fit to the data at all epochs, with an average amount of dust absorption at $\lambda_{\text {rest }}=3000 \AA$ of $A_{3000}=0.25 \pm 0.07$ mag. These results indicate both that the primeval galaxy at $z=6.3$ hosting this GRB has already enriched its ISM with dust, and that it is not possible to characterize the nature of dust suppliers in this source.

\section{Discussion}

The deviation of the extinction curve of high-z quasars from the ones observed in the local Universe may suggest that different dust suppliers are in place at different cosmic epochs, or may be indicative of a different mechanism for processing dust into the ISM. To test the first hypothesis it is necessary to compare the MEC with theoretical models of extinction curves by AGBs, super AGBs and SNe. However, no predictions are available so far for the reddening properties of dust by 


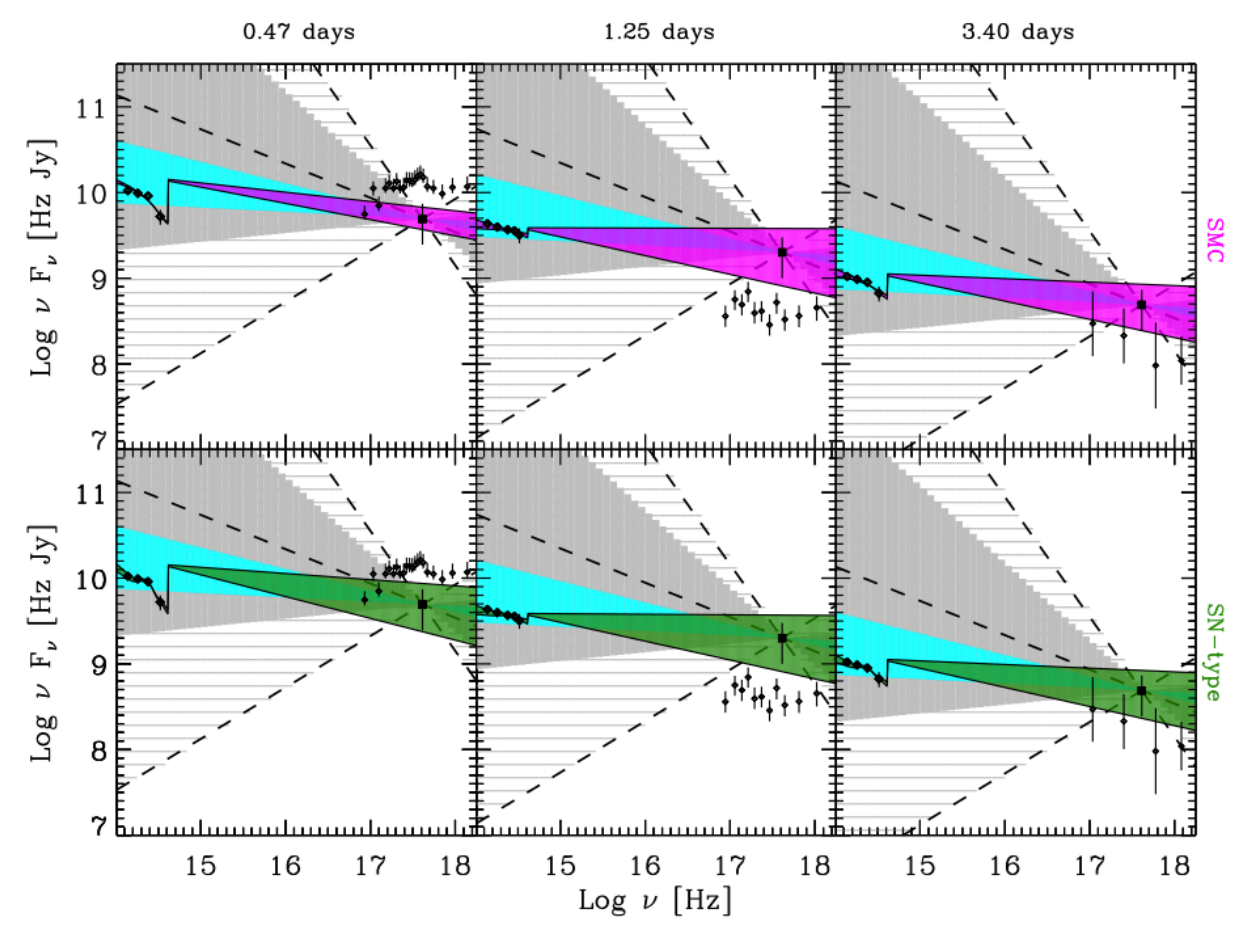

Figure 3: Optical to X-ray SED at $0.47,1.25$ and 3.4 days after the burst trigger (from left to right) and fitted using the two extinction curves that provide excellent fit at all epochs, that is, the SMC and the SNtype (from top to bottom). Squares show the X-ray flux obtained by our analysis while diamonds are the fluxes by Zafar et al. [10]. The hatched area (delimited by dashed lines) indicates the range of intrinsic power-laws consistent with the $\pm 2 \sigma$ uncertainty on the spectral slope $\beta_{X}$. The dashed line in the middle shows the power-law resulting from the best-fit slope to the X-ray data at late epochs $\left(\beta_{X}=1.4\right)$. The gray shaded areas show the range of intrinsic power-laws consistent with the $\pm 1 \sigma$ uncertainty on $\beta_{X}$. The cyan shaded areas show the the power-laws expected from synchrotron emission. The colored areas show the optical to X-ray SED best-fit assuming a dust absorbed power-law model with index free to vary within the range $\beta_{X} \pm 2 \sigma$ (Table 3 ).

these stars. Ventura et al. [23-24], Di Criscienzo et al. [25], and Ventura et al. [26] have computed the composition and grain size distributions of dust produced by stars with masses $0.8<M_{*}<8 M_{\odot}$ at different metallicities $\left(3 \times 10^{-4}<Z<8 \times 10^{-3}\right)$. By starting from these results, to improve the presented analysis, it would be useful to compute the extinction curve of AGB and super AGB stars. These stars are believed to be the dominant stellar sources of dust in the present-day Universe [27] and their contribution to dust enrichment can not be neglected even at redshift $z>6$ [28].

\section{Acknowledgments}

I would like to thank my collaborators that have contributed to the work presented: R. Maiolino, G. Stratta, P. Ventura, M. Di Criscienzo, S. Bianchi, R. Carini, F. D’Antona, F. Dell'Agli, X. Fan, L. Jiang, Y. Juarez, F. La Franca, F. Mannucci, A. Marconi, T. Nagao, T. Oliva, C. Rossi, R. Schneider, A. Tornambé, R. Valiante, C. Willott. 


\section{References}

[1] B. D. Savage and J. S. Mathis, Observed properties of interstellar dust, ARA\&A 21, 177 (1979)

[2] E. L. Fitzpatrick, Interstellar Extinction in External Galaxies, IAU Symp. 135, 37 (1989)

[3] G. T. Richards, et al., Red and Reddened Quasars in the SDSS, AJ 126, 1131 (2003)

[4] P. F. Hopkins et al., Dust Reddening in Sloan Digital Sky Survey Quasars, AJ 128, 1112 (2004)

[5] R. Maiolino et al., A supernova origin for dust in a high-redshift quasar, Nature 431, 533 (2004)

[6] G. Stratta, et al., Dust Properties at $z=6.3$ in the Host Galaxy of GRB 050904, ApJ 661, L9 (2007)

[7] S. Gallerani et al., The extinction law at high redshift and its implications, A\&A 523, 85 (2010)

[8] D. A. Perley, et al., Evidence for supernova-synthesized dust from the rising afterglow of GRB071025 at $z \sim 5$, MNRAS 406, 2473 (2010)

[9] G. Stratta, et al., Is GRB 050904 at z = 6.3 absorbed by dust?, A\&A 532, 45 (2011)

[10] T. Zafar, et al., No evidence for dust extinction in GRB 050904 at $z \sim 6.3$, A\&A 515, 94 (2010)

[11] P. Schady, et al., The dust extinction curves of gamma-ray burst host galaxies, A\&A 537, 15 (2012)

[12] J. Hjorth, et al., On Inferring Extinction Laws in $z \sim 6$ Quasars as Signatures of Supernova Dust, ApJ 768, 173 (2013)

[13] T. A. Reichard, et al., Continuum and Emission-Line Properties of BAL Quasars, AJ 126, 2594 (2003)

[14] Y. C. Pei, Interstellar dust from the Milky Way to the Magellanic Clouds, ApJ 395, 130 (1992).

[15] P. Todini and A. Ferrara, Dust formation in primordial Type II supernovae, MNRAS 325, 726 (2001)

[16] S. Bianchi and R. Schneider, Dust formation and survival in SN ejecta, MNRAS 378, 973 (2007)

[17] H. Hirashita, et al., Extinction curves flattened by reverse shocks in SNe, MNRAS, 384, 1725 (2008)

[18] Y. Juarez et al., The metallicity of the most distant quasars, A\&A 494, L25 (2009)

[19] L. Jiang et al., Probing the Evolution of IR properties of $z \sim 6$ Quasars, AJ 132, 2127 (2006)

[20] C. J. Willott, et al., Four Quasars above Redshift 6 Discovered by the CFHQS, AJ 134, 2435 (2007)

[21] D. J. Mortlock, et al., Properties of Radio-selected Broad Absorption Line Quasars from the First Bright Quasar Survey, A\&A 505, 97 (2009)

[22] R. H. Becker et al., ApJ 538, 72 (2000)

[23] P. Ventura et al., Dust formation around AGB and SAGB stars: a trend with metallicity?, MNRAS 424, 2345 (2012)

[24] P. Ventura et al., The transition from carbon dust to silicate production in low-metallicity asymptotic giant branch and super-asymptotic giant branch stars, MNRAS 420, 1442 (2012)

[25] P. Di Criscienzo et al., Dust formation in the winds of AGBs: the contribution at low metallicities, MNRAS 433, 313 (2013)

[26] P. Ventura et al., Dust from AGB stars: relevant factors and modelling uncertainties, MNRAS 439, 977 (2014)

[27] A. D. Ferrarotti and H. P. Gail, Composition and quantities of dust produced by AGB-stars and returned to the ISM, A\&A 553, 576 (2006)

[28] R. Valiante et al., Stellar sources of dust in the high-redshift Universe, MNRAS 416, 1916 (2011) 